\title{
Finite-time Passivity Control for a Kind of Nonlinear System Based on T-S Fuzzy Model
}

\author{
Enliang Zhao, Lihua Sun ${ }^{*}$, Baoyan Zhu and Chunling Chang \\ School of Science, Shenyang Jianzhu University, Shenyang, China 110168 \\ E-mail: zhaoel88@163.com
}

Keywords: Finite-time Boundedness, passive Control, T-S Fuzzy Model, descriptor system

\begin{abstract}
By means of Lyapunov function and the finite-time theory, the problems of finite-time boundedness and passive control for a kind of nonlinear system described by T-S fuzzy model are studied. The sufficient conditions, which maintain finite-time boundedness and satisfy the passive control performance are by means of designing the controller. The condition is expressed by linear matrix inequalities, and the state feedback gain matrix is obtained by solving these matrix inequalities. Numerical examples illustrate the effectiveness of the proposed method.
\end{abstract}

\section{Introduction}

In practical engineering, people are more interested in the transient stability of the system than Lyapunov's stability. If a stable system has a bad transient performance, it will cause a bad effect on the system and can not meet the application requirements of the industrial process.Finite-time stability describes the system's transient performance, which requires the state response of system is controlled in an ideal area in a fixed finite-time interval. It has very important significance in reality. since Weiss proposed the concept of finite-time stability[1]. Some achievements have been made[2-6].

The concept of passivity was first introduced into control by Lurie and Popov. After the development of Yakubovich, Zames and Moylan, the existing concept of passivity is formed[7]. Passivity is the product of input and output as the rate of energy supply, which reflects the attenuation of energy under the condition of bounded input. Byrnes et al. Combined the passive system theory with the nonlinear geometry theory, and solved the problem that the affine nonlinear system can be equivalent to a passive system through the smooth state feedback[8]. In document [9], the passive control problem of a class of T-S fuzzy descriptor systems is studied. The sufficient and necessary conditions permissible for this kind of system are proved, and the sufficient conditions for the strict passivity of the closed loop system are obtained. At the same time, the sufficient condition for the strict passivity of the closed loop system is obtained. In document [10], the passive control problem of a class of switched generalized systems with nonlinear perturbation term and time delay uncertainty is studied. The sufficient conditions for the asymptotically stable and strict passivity of the nonlinear switched descriptor systems are obtained.

In this paper, we study a finite time passive control problem for a class of T-S fuzzy descriptor systems. By using Lyapunov function and the finite-time theory, the sufficient conditions for the finite-time passive control of a T-S fuzzy descriptor system are proved, and this condition is represented by linear matrix inequalities (LMI). The design method of the controller is given, and a numerical example is given to illustrate the effectiveness of the proposed method.

\section{Problem Statement}

Consider the finite-time passive control problems for a kind of nonlinear descriptor systems via a T-S fuzzy model. The nonlinear dynamical descriptor system can be described by the following fuzzy IF-THEN rules:

$$
R_{i}: \text { if } \theta_{1}(t) \text { is } M_{1 i} \text { and } \theta_{2}(t) \text { is } M_{2 i} \cdots \theta_{p}(t) \text { is } M_{p i}
$$


then $\begin{aligned} & E \dot{x}(t)=A_{i} x(t)+B_{1 i} u(t)+B_{2 i} \omega(t) \\ & z(t)=C_{i} x(t)+D_{i} \omega(t)\end{aligned}$

Where $x(t) \in R^{n}$ is the state, $u(t) \in R^{m}$ is control input; $z(t) \in R^{n}$ is the controlled output. The matrices $A_{i}, C_{i}, B_{1 i}, B_{2 i}, D_{i}$ and are known real constant matrices with appropriate dimensions, $i=1,2, \cdots, r . \omega(t) \in R^{l}$ is the exogenous disturbance and satisfies $\omega^{\mathrm{T}}(t) \omega(t) \leq d$, where $d$ is a positive number.

By using the fuzzy inference method of singleton fuzzifier and weighted average defuzzifier, the overall fuzzy model can be inferred as follows:

$$
\begin{aligned}
& E \dot{x}(t)=\sum_{i=1}^{r} \mu_{i}(\theta(t))\left[A_{i} x(t)+B_{1 i} u(t)+B_{2 i} \omega(t)\right] \\
& z(t)=\sum_{i=1}^{r} \mu_{i}(\theta(t)) C_{i} x(t)+D_{i} \omega(t)
\end{aligned}
$$

For the fuzzy model (2), we consider the following fuzzy controller via the PDC:

$$
u(t)=\sum_{i=1}^{r} \mu_{i}(\theta(t)) K_{i} x(t)
$$

Where $K_{i}$ is local gain matrix . We apply this controller $u(t)$ to system (2) which will result in the following closed-loop systems:

$$
\begin{aligned}
& E \dot{x}(t)=\sum_{i=1}^{r} \sum_{j=1}^{r} \mu_{i}(\theta(t)) \mu_{j}(\theta(t))\left[\left(A_{i}+B_{1 i} K_{j}\right) x(t)+B_{2 i} \omega(t)\right] \\
& z(t)=\sum_{i=1}^{r} \mu_{i}(\theta(t))\left[C_{i} x(t)+D_{i} \omega(t)\right]
\end{aligned}
$$

It is assumed that the system (3) has zero initial conditions $x(0)=0$.

Definition 1: the descriptor system is said to be finite-time boundedness with respect to $\left(c_{1}, c_{2}, T, R, d\right)$.

Where $0<c_{1}<c_{2}$ and $d>0$, if $x^{\mathrm{T}}(0) E^{T} R E x(0) \leq c_{1}$ implies $x^{\mathrm{T}}(t) E^{T} R E x(t) \leq c_{2}, \forall t \in[0, T], \forall \omega(t)$, $\omega^{\mathrm{T}}(t) \omega(t) \leq d$.

Definition 2: The closed loop system (3) is called strictly passive: for a given $\delta>0$, under the zero initial condition $u(t)=0$, for all $\tau>0, \omega(t) \in L_{2}[0, \tau]$, satisfy $V(x(\tau)) \leq \int_{0}^{\tau} \omega^{\mathrm{T}}(t) z(t) \mathrm{d} t-\delta \int_{0}^{\tau} \omega^{\mathrm{T}}(t) \omega(t) \mathrm{d} t, \quad V$ is a continuous nonnegative real value function, which satisfies the $V(0)=0$.

\section{Main Result}

Theorem 1 The descriptor system (3) is said to be finite-time boundedness with respect to $\left(c_{1}, c_{2}, T, R, d\right)$, if there exist nonsingular matrix $P$ and positive definite matrix $\widetilde{P}$, positive numbers $\alpha$ and $\gamma$, such that the following set of matrix inequalities hold:

$$
\begin{aligned}
& P^{\mathrm{T}} E=E^{\mathrm{T}} P \geq 0 \\
& P^{\mathrm{T}} E=E^{\mathrm{T}} R^{\frac{1}{2}} \widetilde{P} R^{\frac{1}{2}} E \\
& {\left[\begin{array}{cc}
\Pi_{1} & P^{T} B_{2 i} \\
* & -\gamma^{2} I
\end{array}\right]<0 \quad i=1,2, \cdots, r}
\end{aligned}
$$




$$
\begin{aligned}
& {\left[\begin{array}{cc}
\Pi_{2} & P^{T}\left(B_{2 i}+B_{2 j}\right) \\
* & -2 \gamma^{2} I
\end{array}\right]<0 \quad i<j \leq r} \\
& \frac{e^{\alpha T}\left(\lambda_{\max }(\widetilde{P}) C_{1}+d \gamma^{2} T\right)}{\lambda_{\min }(\widetilde{P})}<c_{2}
\end{aligned}
$$

Where $\Pi_{1}=\left(A_{i}^{\mathrm{T}}+K_{i}^{T} B_{1 i}^{T}\right) P+P^{T}\left(A_{i}+B_{1 i} K_{i}\right)-\alpha E^{T} P$,

$$
\Pi_{2}=\left(A_{i}^{\mathrm{T}}+A_{j}^{\mathrm{T}}+K_{i}^{T} B_{1 j}^{T}+K_{j}^{T} B_{1 i}^{T}\right) P+P^{T}\left(A_{i}+A_{j}+B_{1 i} K_{j}+B_{1 j} K_{i}\right)-2 \alpha E^{T} P
$$

Proof. Let $V(x(t))=x^{\mathrm{T}}(t) E^{T} P x(t)$, then $\dot{V}(x(t))=\dot{x}^{T}(t) E^{T} P x(t)+x^{T}(t) P^{T} E \dot{x}(t)$

$$
\begin{gathered}
=\sum_{i=1}^{r} \sum_{j=1}^{r} \mu_{i}(\theta(t)) \mu_{j}(\theta(t))\left[\left(A_{i}+B_{1 i} K_{j}\right) x(t)+B_{2 i} \omega(t)\right]^{T} P x(t) \\
+x^{T}(t) P^{T} \sum_{i=1}^{r} \sum_{j=1}^{r} \mu_{i}(\theta(t)) \mu_{j}(\theta(t))\left[\left(A_{i}+B_{1 i} K_{j}\right) x(t)+B_{2 i} \omega(t)\right] \\
=\sum_{i=1}^{r} \sum_{j=1}^{r} \mu_{i}(\theta(t)) \mu_{j}(\theta(t))\left(\begin{array}{c}
x(t) \\
\omega(t)
\end{array}\right)^{\mathrm{T}}\left(\begin{array}{cc}
\left(A_{i}^{\mathrm{T}}+K_{j}^{T} B_{1 i}^{T}\right) P+P^{T}\left(A_{i}+B_{1 i} K_{j}\right) & P^{T} B_{2 i} \\
* & 0
\end{array}\right)\left(\begin{array}{c}
x(t) \\
\omega(t)
\end{array}\right) .
\end{gathered}
$$

It follows from (6) and (7) in Theorem 1 that

$$
\dot{V}(x(t))-\alpha V(x(t))-\gamma^{2} \omega^{T} \omega<0
$$

Before and after multiplying (9) by $e^{-\alpha t}$, then integrating the inequality form 0 to $T$ leads to $e^{-\alpha t} V(x(t))-V(x(0))<\gamma^{2} d T$

From (5),

$$
\begin{aligned}
& V(x(t))=x^{T}(t) E^{T} R^{\frac{1}{2}} \widetilde{P} R^{\frac{1}{2}} \operatorname{Ex}(t) \geq \lambda_{\text {min }}(\widetilde{P}) x^{T}(t) E^{T} R E x(t) \\
& V(x(0))=x^{T}(0) E^{T} R^{\frac{1}{2}} \widetilde{P} R^{\frac{1}{2}} \operatorname{Ex}(0) \leq \lambda_{\text {max }}(\widetilde{P}) x^{T}(0) E^{T} R E x(0)
\end{aligned}
$$

By substituting (11) and (12) into (10), based on (8), we obtain the inequality

$$
x^{T}(t) E^{T} R E x(t) \leq \frac{e^{\alpha T}\left(\lambda_{\max }(\widetilde{P}) c_{1}+\gamma^{2} d T\right)}{\lambda_{\text {min }}(\widetilde{P})}<c_{2}
$$

System (3) is finite-time bounded with respect to $\left(c_{1}, c_{2}, T, R, d\right)$.

In the follow theorem, we transform the matrix inequalities in Theorem 1 into LMI.

Theorem 2 The descriptor system (3) is said to be finite-time boundedness with respect to $\left(c_{1}, C_{2}, T, R, d\right)$, if there exist nonsingular matrix $M$ and positive definite matrix $\widetilde{P}$, positive numbers $\alpha$ and $\gamma$, such that the following set of matrix inequalities hold:

$$
\begin{aligned}
& E M^{\mathrm{T}}=M E^{\mathrm{T}} \geq 0 \\
& E=M^{\mathrm{T}} E^{\mathrm{T}} R^{\frac{1}{2}} \widetilde{P} R^{\frac{1}{2}} E \\
& {\left[\begin{array}{cc}
\Pi_{3} & B_{2 i} \\
* & -\gamma^{2} I
\end{array}\right]<0 \quad i=1,2, \cdots, r} \\
& {\left[\begin{array}{cc}
\Pi_{4} & B_{2 i}+B_{2 j} \\
* & -2 \gamma^{2} I
\end{array}\right]<0 \quad i<j \leq r} \\
& \frac{e^{\alpha T}\left(\lambda_{\max }(\widetilde{P}) c_{1}+d \gamma^{2} T\right)}{\lambda_{\min }(\widetilde{P})}<c_{2}
\end{aligned}
$$

Where $\Pi_{3}=M A_{i}^{T}+X_{j}^{T} B_{1 i}^{T}+A_{i} M^{T}+B_{1 i} X_{j}-\alpha M E^{T}$,

$$
\Pi_{4}=M\left(A_{i}^{\mathrm{T}}+A_{j}^{\mathrm{T}}\right)+X_{i}^{T} B_{1 j}^{T}+X_{j}^{T} B_{1 i}^{T}+\left(A_{i}+A_{j}\right) M^{T}+B_{1 i} X_{j}+B_{1 j} X_{i}-2 \alpha M E^{T}
$$


Proof. Pre- and post- multiplying (4) by $P^{-T}$ and $P^{-1}$, pre- and post- multiplying (6) and (7) by $\left[\begin{array}{cc}P^{-T} & 0 \\ 0 & I\end{array}\right]$ and $\left[\begin{array}{cc}P^{-1} & 0 \\ 0 & I\end{array}\right]$, let $M=P^{-T}, X_{i}=K_{i} M^{T}, X_{j}=K_{j} M^{T}$, we can get (13), (15) and (16).

Remark. The existing literature is the formula (17) into an equivalent linear inequality form. In fact, if known $\left(c_{1}, c_{2}, T, R, d\right)$, formula (13) - (17) can be solved directly in the LMI toolbox.

Theorem 3. The descriptor system (3) is said to be passive and finite-time boundedness with respect to $\left(c_{1}, c_{2}, T, R, d\right)$, if there exist nonsingular matrix $P$ and positive definite matrix $\widetilde{P}$, positive numbers $\alpha, \gamma$ and $\delta$, such that the following set of matrix inequalities hold:

$$
\begin{aligned}
& P^{\mathrm{T}} E=E^{\mathrm{T}} P \geq 0 \\
& P^{\mathrm{T}} E=E^{\mathrm{T}} R^{\frac{1}{2}} \widetilde{P} R^{\frac{1}{2}} E \\
& {\left[\begin{array}{cc}
\Pi_{1} & P^{T} B_{2 i} \\
* & -\gamma^{2} I
\end{array}\right]<0 \quad i=1,2, \cdots, r} \\
& {\left[\begin{array}{cc}
\Pi_{2} & P^{T}\left(B_{2 i}+B_{2 j}\right) \\
* & -2 \gamma^{2} I
\end{array}\right]<0 \quad i<j \leq r} \\
& \delta I-D_{i}^{\mathrm{T}}<0 \\
& {\left[\begin{array}{cc}
\Pi_{5} & P^{\mathrm{T}} B_{2 i}-\frac{1}{2} C_{i}^{\mathrm{T}} \\
* & \delta I-D_{i}^{\mathrm{T}}
\end{array}\right]<0 \quad i=1,2, \cdots, r} \\
& 2 \delta I-D_{i}^{\mathrm{T}}-D_{j}^{\mathrm{T}}<0 \\
& {\left[\begin{array}{cc}
\Pi_{6} & P^{\mathrm{T}}\left(B_{2 i}+B_{2 j}\right)-\frac{1}{2} C_{i}^{\mathrm{T}}-\frac{1}{2} C_{j}^{\mathrm{T}} \\
* & 2 \delta I-D_{i}^{\mathrm{T}}-D_{j}^{\mathrm{T}}
\end{array}\right]<0 \quad i<j \leq r} \\
& \frac{e^{\alpha T}\left(\lambda_{\max }(\widetilde{P}) C_{1}+d \gamma^{2} T\right)}{\lambda_{\min }(\widetilde{P})}<C_{2}
\end{aligned}
$$

Where

$$
\begin{aligned}
& \Pi_{1}=\left(A_{i}^{\mathrm{T}}+K_{i}^{T} B_{1 i}^{T}\right) P+P^{T}\left(A_{i}+B_{1 i} K_{i}\right)-\alpha E^{T} P, \\
& \Pi_{2}=\left(A_{i}^{\mathrm{T}}+A_{j}^{\mathrm{T}}+K_{i}^{T} B_{1 j}^{T}+K_{j}^{T} B_{1 i}^{T}\right) P+P^{T}\left(A_{i}+A_{j}+B_{1 i} K_{j}+B_{1 j} K_{i}\right)-2 \alpha E^{T} P, \\
& \Pi_{5}=A_{i}^{\mathrm{T}} P+P^{\mathrm{T}} A_{i}+K_{i}^{\mathrm{T}} B_{1 i}^{\mathrm{T}} P+P^{\mathrm{T}} B_{1 i} K_{i}, \\
& \Pi_{6}=A_{i}^{\mathrm{T}} P+P^{\mathrm{T}} A_{i}+K_{j}^{\mathrm{T}} B_{1 i}^{\mathrm{T}} P+P^{\mathrm{T}} B_{1 i} K_{j}+A_{j}^{\mathrm{T}} P+P^{\mathrm{T}} A_{j}+K_{i}^{\mathrm{T}} B_{1 j}^{\mathrm{T}} P+P^{\mathrm{T}} B_{1 j} K_{i}
\end{aligned}
$$

Proof. Based on Theorem 1, the system is finite-time boundedness with respect to $\left(c_{1}, C_{2}, T, R, d\right)$ from (18)--(21) and (26), now we will prove that the system (3) is passivity.

Let $V(x(t))=x^{\mathrm{T}}(t) E^{T} P x(t)$, under the premise of the establishment of (22) and (24), according to (23) and (25), then

$$
\begin{aligned}
& \dot{V}(x(t))-z^{\mathrm{T}}(t) \omega(t)+\delta \omega^{\mathrm{T}}(t) \omega(t) \\
& =\sum_{i=1}^{r} \sum_{j=1}^{r}\left(\begin{array}{c}
x \\
\omega
\end{array}\right)\left(\begin{array}{cc}
A_{i}^{\mathrm{T}} P+P^{\mathrm{T}} A_{i}+K_{j}^{\mathrm{T}} B_{1 i}^{\mathrm{T}} P+P^{\mathrm{T}} B_{1 i} K_{j}+C_{i}^{\mathrm{T}} C_{i} & P^{\mathrm{T}} B_{2 i}-\frac{1}{2} C_{i}^{\mathrm{T}} \\
* & \delta I-D_{i}^{\mathrm{T}}
\end{array}\right)\left(\begin{array}{l}
x \\
\omega
\end{array}\right)<0
\end{aligned}
$$

From the upper and attention to $V(x(0))=0$, then

$$
V(x(\tau)) \leq \int_{0}^{\tau} \omega^{\mathrm{T}}(t) z(t) \mathrm{d} t-\delta \int_{0}^{\tau} \omega^{\mathrm{T}}(t) \omega(t) \mathrm{d} t .
$$

Therefore, the system (3) is passive. 
Theorem 4. The descriptor system (3) is said to be passive and finite-time boundedness with respect to $\left(c_{1}, c_{2}, T, R, d\right)$, if there exist nonsingular matrix $M$ and positive definite matrix $\widetilde{P}$, positive numbers $\alpha, \gamma$ and $\delta$, such that the following set of matrix inequalities hold:

$$
\begin{aligned}
& E M^{\mathrm{T}}=M E^{\mathrm{T}} \geq 0 \\
& E=M E^{\mathrm{T}} R^{\frac{1}{2}} \widetilde{P} R^{\frac{1}{2}} E \\
& {\left[\begin{array}{cc}
\Pi_{3} & B_{2 i} \\
* & -\gamma^{2} I
\end{array}\right]<0 \quad i=1,2, \cdots, r} \\
& {\left[\begin{array}{cc}
\Pi_{4} & B_{2 i}+B_{2 j} \\
* & -2 \gamma^{2} I
\end{array}\right]<0 \quad i<j \leq r} \\
& \delta I-D_{i}^{\mathrm{T}}<0 \\
& {\left[\begin{array}{cc}
\Pi_{7} & B_{2 i}-\frac{1}{2} M C_{i}^{\mathrm{T}} \\
* & \delta I-D_{i}^{\mathrm{T}}
\end{array}\right]<0 \quad i=1,2, \cdots, r} \\
& 2 \delta I-D_{i}^{\mathrm{T}}-D_{j}^{\mathrm{T}}<0 \\
& {\left[\begin{array}{cc}
\Pi_{8} & B_{2 i}+B_{2 j}-\frac{1}{2} M C_{i}^{\mathrm{T}}-\frac{1}{2} M C_{j}^{\mathrm{T}} \\
* & 2 \delta I-D_{i}^{\mathrm{T}}-D_{j}^{\mathrm{T}}
\end{array}\right]<0 \quad i<j \leq r} \\
& \frac{e^{\alpha \mathrm{T}}\left(\lambda_{\max }(\widetilde{P}) C_{1}+d \gamma^{2} T\right)}{\lambda_{\min }(\widetilde{P})}<C_{2}
\end{aligned}
$$

where $\Pi_{3}=M A_{i}^{\mathrm{T}}+X_{i}^{T} B_{1 i}^{T}+A_{i} M^{T}+B_{1 i} X_{i}-\alpha M E^{T}$,

$$
\begin{aligned}
& \Pi_{4}=M\left(A_{i}^{\mathrm{T}}+A_{j}^{\mathrm{T}}\right)+X_{i}^{T} B_{1 j}^{T}+X_{j}^{T} B_{1 i}^{T}+\left(A_{i}+A_{j}\right) M^{T}+B_{1 i} X_{j}+B_{1 j} X_{i}-2 \alpha M E^{T}, \\
& \Pi_{7}=M A_{i}^{\mathrm{T}}+A_{i} M^{\mathrm{T}}+X_{i}^{\mathrm{T}} B_{1 i}^{\mathrm{T}}+B_{1 i} X_{i}, \\
& \Pi_{8}=M A_{i}^{\mathrm{T}}+A_{i} M^{\mathrm{T}}+X_{j}^{\mathrm{T}} B_{1 i}^{\mathrm{T}}+B_{1 i} X_{j}+M A_{j}^{\mathrm{T}}+A_{j} M^{\mathrm{T}}+X_{i}^{\mathrm{T}} B_{1 j}^{\mathrm{T}}+B_{1 j} X_{i} .
\end{aligned}
$$

Proof. Pre- and post- multiplying (18) by $P^{-T}$ and $P^{-1}$, pre- and post- multiplying (20) , (21), (23) and(25) by $\left[\begin{array}{ll}P^{-T} & \\ & I\end{array}\right]$ and $\left[\begin{array}{rr}P^{-1} & \\ & I\end{array}\right]$ let $M=P^{-T}, X_{i}=K_{i} M^{T}, X_{j}=K_{j} M^{T}$, we can get (27),(29),(30),(32) and (34).

\section{Numerical Example}

T-S fuzzy nonlinear systems are considered as follows:

$R_{i}$ :if $\theta_{1}(t)$ is $M_{1 i}$

$$
\text { then } \begin{cases}E \dot{x}(t)=\sum_{i=1}^{r} \sum_{j=1}^{r} \mu_{i}(\theta(t)) \mu_{j}(\theta(t))\left[\left(A_{i}+B_{1 i} K_{j}\right) x(t)+B_{2 i} \omega(t)\right] & \\ z(t)=\sum_{i=1}^{r} \mu_{i}(\theta(t))\left[C_{i} x(t)+D_{i} \omega(t)\right] & i=1,2\end{cases}
$$

Its normalization membership functions are as follows: $u_{1}\left(x_{1}(t)\right)=\frac{1}{2}\left(1-\frac{x_{1}(t)}{t}\right)$,

$u_{2}\left(x_{1}(t)\right)=\frac{1}{2}\left(1+\frac{x_{1}(t)}{t}\right)$, state variable $x(t)=\left[x_{1}(t), x_{2}(t), x_{3}(t)\right]^{\mathrm{T}}$ in which $x_{i}(t) \in R^{1}, i=1,2,3$, take the following control parameters: 


$$
\begin{aligned}
& E=\left(\begin{array}{lll}
1 & 0 & 0 \\
0 & 1 & 0 \\
0 & 0 & 1
\end{array}\right) \\
& R=\left(\begin{array}{lll}
1 & 0 & 0 \\
0 & 1 & 0 \\
0 & 0 & 1
\end{array}\right) \\
& A_{1}=\left(\begin{array}{lll}
-0.1107 & -0.0610 & 0.0361 \\
0.0972 & 0.0851 & -0.0893 \\
-0.1186 & -0.1331 & 0.1917
\end{array}\right), A_{2}=\left(\begin{array}{ccc}
0.0261 & 0.0871 & -0.1540 \\
-0.1860 & 0.1202 & 0.1457 \\
-0.0993 & 0.1021 & -0.0807
\end{array}\right) \text {, } \\
& B_{11}=\left(\begin{array}{l}
0.0706 \\
0.1152 \\
-0.0085
\end{array}\right), B_{12}=\left(\begin{array}{l}
-0.1889 \\
-0.0705 \\
-0.1822
\end{array}\right), B_{21}=\left(\begin{array}{lll}
1 & 0 & 0 \\
0 & 1 & 0 \\
0 & 0 & 0
\end{array}\right), B_{22}=\left(\begin{array}{lll}
1 & 0 & 0 \\
0 & 1 & 0 \\
0 & 0 & 0
\end{array}\right) \text {, } \\
& C_{1}=\left(\begin{array}{ccc}
-0.1732 & 0.1509 & 0.0628 \\
-0.1553 & -0.0630 & -0.1159 \\
-0.0813 & 0.1817 & 0.1248
\end{array}\right), C_{2}=\left(\begin{array}{ccc}
0.0464 & 0.0975 & -0.0570 \\
0.1652 & -0.1547 & -0.0331 \\
-0.1675 & 0.0184 & -0.1493
\end{array}\right) \text {, } \\
& D_{1}=\left(\begin{array}{lll}
1 & 0 & 0 \\
0 & 1 & 0 \\
0 & 0 & 0
\end{array}\right), D_{2}=\left(\begin{array}{lll}
1 & 0 & 0 \\
0 & 1 & 0 \\
0 & 0 & 0
\end{array}\right) \text {, } \\
& c_{1}=2, c_{2}=10, d=0.0732, T=2
\end{aligned}
$$

According to theorem 4, let $\alpha=0.01$,Using the LMI toolbox, we can see that inequalities (27)-(35) can be solved, The feasible solution is as follows:

$$
\begin{aligned}
& M=\left(\begin{array}{ccc}
11.7760 & 24.6108 & -33.6762 \\
24.6108 & 59.8942 & 9.2535 \\
0 & 0 & -7.8616
\end{array}\right), \\
& \widetilde{P}=\left(\begin{array}{ccc}
11.7760 & 24.6108 & 0 \\
24.6108 & 59.8942 & 0 \\
0 & 0 & 11.7760
\end{array}\right), \\
& \gamma=0.0719 \text { and } \delta=0.0349 .
\end{aligned}
$$

So the controller is:

$$
\begin{aligned}
& K_{1}=(425.7732-177.4549-15.4421) \text {, } \\
& K_{2}=1.0 \mathrm{e}+003 *(1.1043 \quad-0.4623-0.0200) \text {. }
\end{aligned}
$$

So the state feedback controller is:

$$
u(t)=\left\{\frac{1}{2}\left(1-\frac{x_{1}(t)}{t}\right)\left(\begin{array}{lll}
1166.6 & -292.8 & 121.4
\end{array}\right)+\frac{1}{2}\left(1+\frac{x_{1}(t)}{t}\right)\left(\begin{array}{lll}
2299.4 & -697.1 & 157.1
\end{array}\right)\right\}
$$

Thus the system (3) is strictly passive. Let $\omega(t)=\left(\begin{array}{lll}e^{-0.9 t} \sin (-0.9 t) & e^{-0.9 t} \cos (-0.9 t) & 1\end{array}\right)^{\mathrm{T}}$. Then the trajectories of $x^{T}(t) E^{T} E x(t)$ for the approximated nonlinear system are shown in Figure 1. 


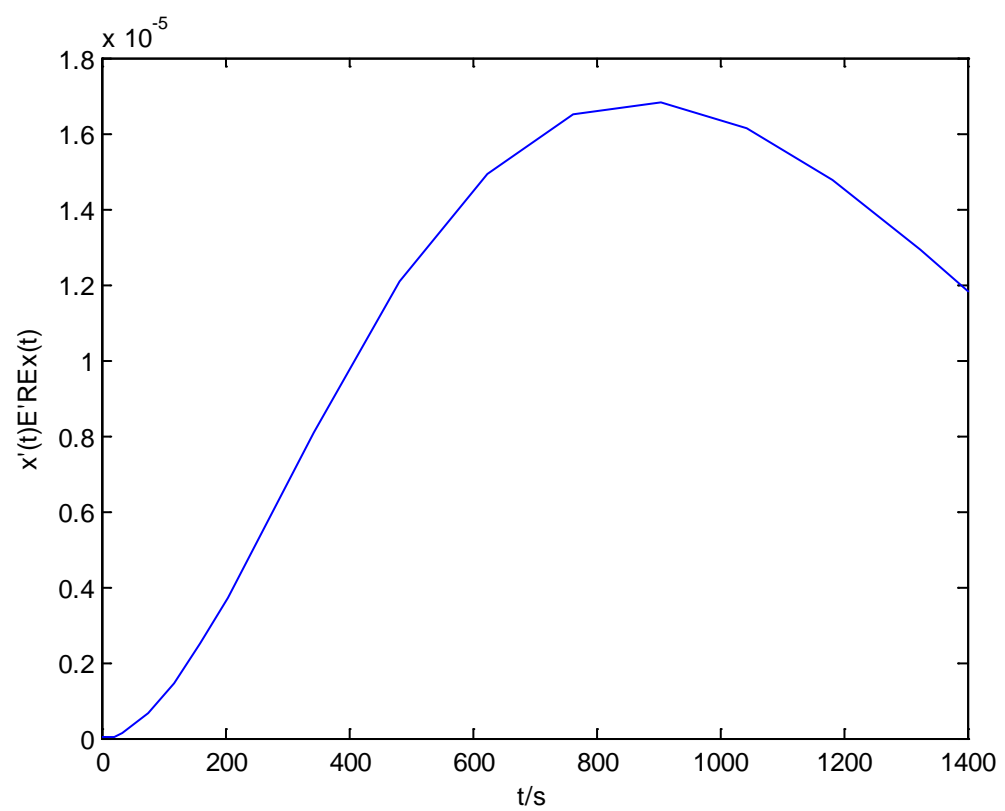

Fig 1: The trajectory of $x^{T}(t) E^{T} E x(t)$ for the nonlinear system approximated by the fuzzy system

\section{Summary}

This paper investigates the problems of finite-time bounds and passive control for a kind of nonlinear descriptor system described by T-S fuzzy model by means of Lyapunov function and the finite-time theory, and provides the sufficient conditions of finite-time bounds and the method of designing the controller. Numerical examples illustrate the effectiveness of the proposed method.

\section{References}

[1] L. Weiss, E. Infante, On the stability of systems defined over a finite time interval. Proceedings of the National Academy of Sciences of the United States of America, (1965)44-48.

[2] F. Amato, M. Ariola, P. Dorato, Finite-time control of linear systems subject to parametric uncertainties and disturbances, Automatica,37(9):1459-1463(2001).

[3] F. Amato, M. Ariola, Finite-time control of discrete-time linear systems, IEEE Transactions on Automatic Control,50(5):724-729(2005).

[4] J.E. Feng, W. Zhen, J.B. Sun. Finite-time control of linear singular system with parametric uncertainties and disturbances, Acta Automatica Sinica,31(4):634-637(2005).

[5] J.R. Liang, C. Zhai, H.L. Wei. Finite-time ${ }^{H_{\infty}}$ control for uncertain singular system, Control Engineering of China, 21(2):241-244(2014).

[6] B.Y. Zhu, J.J. Zhang, D.Q. Zhang, A.B. Liu. Delay-dependent finite-time $H_{\infty}$ controller design for a kind of nonlinear descriptor systems via a T-S fuzzy model, 1-14(2015).

[7] C.B.Feng, K.J.Zhang. Robust control of nonlinear systems , Science Press, Beijing,2004.

[8] Byrnes C I, Isidori A, Willems J C., Passivity,feedback equivalence,and the global stabilization of minimum phase nonlinear systems.IEEE Transactions on Automatic Control, 36(11): 1228-1240(1991).

[9] B.Y. Zhu, Q.L. Zhang, S.C.Tong. The Passivity Control for a Kind of T-S Fuzzy Descriptor System . Theory and practice of system engineering.6:81-87(2006).

[10] S.S.Chen, D.M.Yang. Robust Passive Control for Nonlinear Uncertain Switched Singular Systems with Time-delay[J], Computer Technology and Automation, 36(2):1-4(2017). 